\title{
INTERACTIVE IMMERSIVE VIRTUAL MUSEUM: DIGITAL DOCUMENTATION FOR VIRTUAL INTERACTION
}

\author{
P. Clini ${ }^{\text {a }}$ L. Ruggeri ${ }^{\text {a }}$, R. Angeloni ${ }^{a}$, M. Sasso ${ }^{b}$ \\ ${ }^{a}$ DICEA, Dept. of Civil and Building Engineering and Architecture, Polytechnic University of Marche, Ancona, Italy \\ ${ }^{\mathrm{b}}$ Ubisive, Civitanova Marche, Italy \\ p.clini@univpm.it, (1.ruggeri, r.angeloni)@pm.univpm.it, michele.sasso@ubisive.it
}

Commission II, WG II/8

KEY WORDS: immersive virtual reality, museum technologies, reality based modelling, virtual reconstruction, structure from motion

\begin{abstract}
:
Thanks to their playful and educational approach Virtual Museum systems are very effective for the communication of Cultural Heritage. Among the latest technologies Immersive Virtual Reality is probably the most appealing and potentially effective to serve this purpose; nevertheless, due to a poor user-system interaction, caused by an incomplete maturity of a specific technology for museum applications, it is still quite uncommon to find immersive installations in museums.

This paper explore the possibilities offered by this technology and presents a workflow that, starting from digital documentation, makes possible an interaction with archaeological finds or any other cultural heritage inside different kinds of immersive virtual reality spaces. Two different cases studies are presented: the National Archaeological Museum of Marche in Ancona and the 3D reconstruction of the Roman Forum of Fanum Fortunae. Two different approaches not only conceptually but also in contents; while the Archaeological Museum is represented in the application simply using spherical panoramas to give the perception of the third dimension, the Roman Forum is a 3D model that allows visitors to move in the virtual space as in the real one.

In both cases, the acquisition phase of the artefacts is central; artefacts are digitized with the photogrammetric technique Structure for Motion then they are integrated inside the immersive virtual space using a PC with a HTC Vive system that allows the user to interact with the 3D models turning the manipulation of objects into a fun and exciting experience.

The challenge, taking advantage of the latest opportunities made available by photogrammetry and ICT, is to enrich visitors' experience in Real Museum making possible the interaction with perishable, damaged or lost objects and the public access to inaccessible or no longer existing places promoting in this way the preservation of fragile sites.
\end{abstract}

\section{INTRODUCTION}

The work in this paper, exploring the possibilities offered by modern technology, presents a workflow that starting from the acquisition and modelling of cultural heritage, contents and container, aims to increase access and interaction with artefacts overcoming distance and time.

Thanks to the progresses achieved in the field of 3D surveying techniques is possible to obtain detailed and photorealistic 3D digital representations useful not only for documentation or archaeological and architectural analyses but also for divulgation and educational purpose inside museum exhibitions or exploiting virtual tourism potentialities. However, it is necessary to consider that a museum has a very high number of items so their digitization could take a lot of time. In order to overcome the difficulty of acquire a mass of finds the acquisition phase has been optimized and made expeditious in order also to reduce file size and computation time. The choice of photogrammetry for the digitization of artefacts and the construction of digital 3D models made it possible also obtaining good textures important for the fruition.

The definition of a virtual space where items are displaced is as important as items themselves. Here are presented two different case studies: the National Archaeological Museum of Marche in Ancona and the 3D reconstruction of the Roman Forum of Fanum Fortunae.

The first application, based on a real museum, wants to overcome the idea of the archaeological museum as a site linked to the past, not active, "static", permitting the interaction with the archaeological finds usually "silent" in their display cases.
The second one, concerning an archaeological site, let the user visit a virtual reconstruction of an historical place no longer existing, replacing the real world with a virtual one.

Modern technology, using different types of devices and levels of immersion and interaction, makes possible the combining of education and entertainment, so visitors may discover new contents in a playful manner. The choice of HTC Vive system made possible the integration of 3D models inside the immersive virtual space.

Using such an interactive solution changes visitors' experience; visitors are no more passive spectators but become capable of acting with the $3 \mathrm{D}$ virtual object in its digital three-dimensional physicality.

The workflow includes three phases (Figure 1):

1. acquisition and modelling;

2. optimization of geometries and texture;

3. integration of container and contents for interactive fruition into immersive application.

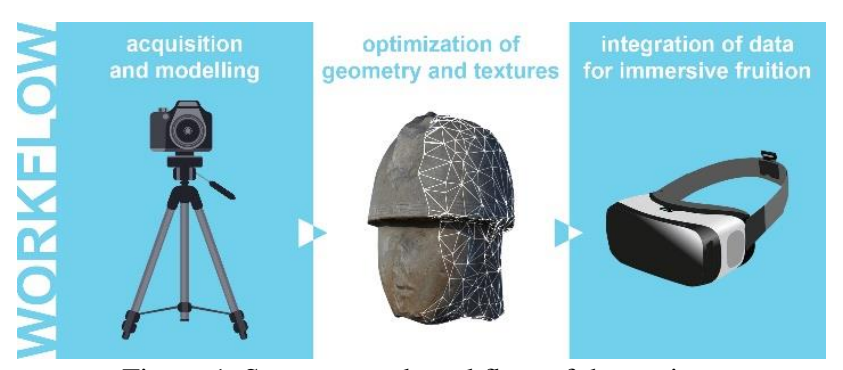

Figure 1. Structure and workflow of the project. 
Therefore, the main goals of this work are:

1. enrichment of visitors' experience in Real Museum implementing the paradigm of "learning by interacting" with innovative Virtual Reality technologies;

2. to allow the visitor to interact with perishable, damaged or lost objects, to make possible public access to inaccessible or no longer existing places promoting in this way the preservation of fragile sites;

3. to enhance the enjoyment of archaeological heritage whose observation with the naked eye inside of the showcases severely limits their value and the knowledge of the details.

4. to define a pipeline that starting from an expeditious and low cost acquisition leads to optimized 3D models for immersive application in the field of fruition.

\section{STATE OF ART}

Cultural organisations and museums are taking advantage of modern technologies in order to offer a more appealing experience to visitors. Nevertheless, Virtual Museums' large spread in many cases has nothing to do with 3D virtual spaces and 3D models (Skamantzari and Georgopoulos, 2016).

Nowadays the development in the field of 3D surveys, also combining different techniques (Dostal and Yamafune, 2018), makes possible the creation of detailed and photo-realistic 3D models useful not only for documentation or for archaeological and architectural analyses but also for divulgation and educational purpose.

Nowadays the potentialities of image-based 3D reconstruction are given. Several studies evidence the reliability, accuracy and flexibility of these techniques but also some lacks in the collection of high number of items especially considering difficulties of digitization campaign like available time, lighting conditions, equipment transport, budget, etc. (Gattet et al., 2015). (Evgenikou and Georgopoulos, 2015) shows different methodology for the three dimensional reconstruction of small artefacts. The accuracy of Structure From Motion technique (SFM), also combined with Focus Stacking for the 3D digitization of small archaeological finds, is verified in (Clini et al., 2016) by comparison with 3D model obtained through triangulation laser scanning.

For what concern the $3 \mathrm{D}$ virtual space, theoretically and methodologically based on (EPOCH, 2009) and (LopezMenchero and Grande, 2011), the reconstruction of lost archaeological architectures has to be developed in different phases starting from the collection and analysis of the sources up to the creation of the reconstructive 3D model in line with the interpretation process (Demetrescu et al., 2016).

An accurate and well-defined acquisition and interpretation of data is a fundamental task but also their communication and dissemination has to be taken into account at the same level.

The development of new ICT (Information and Communication Technology) guarantees the possibility of managing complex reality-based models in real-time so Virtual 3D reconstruction can be used in order to create another reality (Boas, 2013) which replaces the real world making possible an interaction in a totally immersive state projecting also real movements into the virtual world (Anthes et al., 2016).

(Jiménez Fernández-Palacios et al., 2017) demonstrates the importance of the use of ICT technologies to make culture accessible to mass audience.

Museums are themselves technologies of representation and at the same time contexts where technologies of representation are employed or employable (Allen and Lupo, 2012), it is a fact that they need accessible and attractive ways to represent, manage and exploit $\mathrm{CH}$ data at different levels.

\section{ACQUISITION AND MODELING}

The workflow is based on the acquisition and reconstruction of archaeological finds and sites or museums carried out with a special attention for the subsequent fruition. Space and items are then integrated and made available for the final users thanks to a VR system that allows the user to move in the virtual space and to interact with the 3D models taking them out from their display cases, handling and looking artefacts models up close.

\subsection{CRP for artefacts digitization}

Nowadays different 3D recording techniques make possible the generation of 3D models extremely accurate normally consisting of millions of polygons and high-resolution images. Considering that in an archaeological museum there are a lot of artefacts the acquisition phase must be optimized and made expeditious in order to reduce the subsequent times of data processing. The use of Close Range Photogrammetry (CRP), guarantees that requirements and also an excellent photographic quality. The goal of 3D digitization in this study is in facts to obtain a very accurate digital facsimile not only to improve and to enrich the historical and archaeological knowledge about the artefacts but also considering the final aim of a virtual reality fruition.

The CRP pipeline is realized with the acquisition phase, the images orientation and the dense point cloud building (Figure 2).

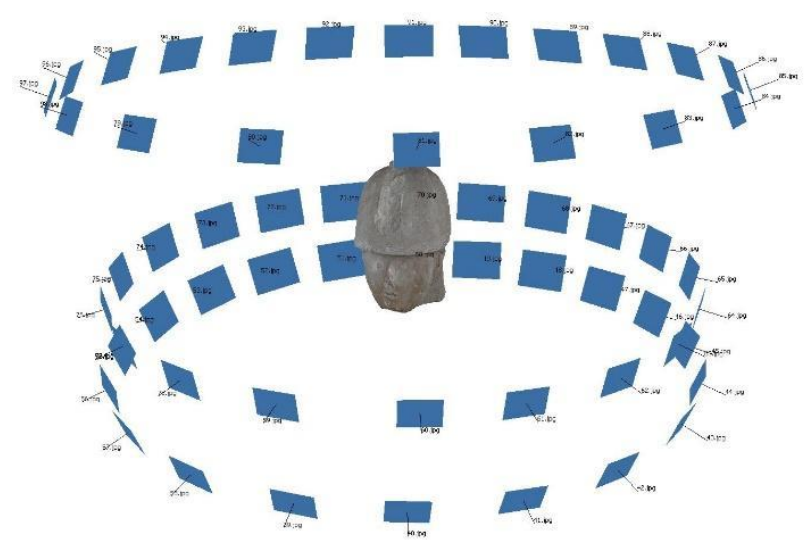

Figure 2. Camera positions and orientation after the alignment phase of the Structure from Motion Pipeline

Depending on the geometry and the size different attempt are necessary to obtain a right balance between accuracy and file size. Particularly important is also the texture, fundamental in the visualization of the artefacts.

Considering the location of the artefacts and their average properties the quick and low cost acquisition system used is composed of a white photographic box, two lamps and a turntable therefore calibrated to medium-small sized items movable from their location.

In order to not create sharp shadows on the artefact the archaeological find to be digitized is placed inside the photographic box that spreads the light of the two lamps. The box is used also to facilitate the next digital reconstruction; no space and object around make it more quick and easy.

Once the project of acquisition is made, the use of a turntable allows rotating the artefact between two consecutive pictures keeping the camera fixed. The rotation is controlled thanks to a graduated scale useful to measure the angle of rotations between pictures. Two metric scales, useful to orient and scale the final 
model, and a colorchecker, for the white balance and the calibration of the camera colours, are also present. 3D models will be fruited in 1:1 scale so is essential in the project phase to consider that. The expected scale depends on the distance of the camera from the artefact, on the focal length used and on the dimension of the pixel, so on the characteristics of the sensor of the camera. It is fundamental to determine the Ground Sample Distance (GSD) that is to say the distance between two consecutive pixel centres measured on the artefact. After deciding the acquisition distance and the focal length to obtain a certain GSD the next step is to decide the number of shots necessary to cover all the artefact and thus obtain a final 3D model complete in all its parts so how many degrees the turntable will be rotated between to consecutive picture and at what heights we put the camera.

To reconstruct a good quality model by using SFM a large number of data is needed but the use of too many pictures is often useless and always leads to long calculation times. Knowing that an overlap of about $60 \%$ between two consecutive pictures is needed the acquisition phase is optimized calculating the angle of rotation required. Particular attention has to be paid on concaves or convex parts that could create shadow areas that must be covered in order to obtain a complete model. Therefore, vertical movement of the camera between subsequent revolutions around the same artefact has to be chosen depending on the morphology of the object (Figure 3).
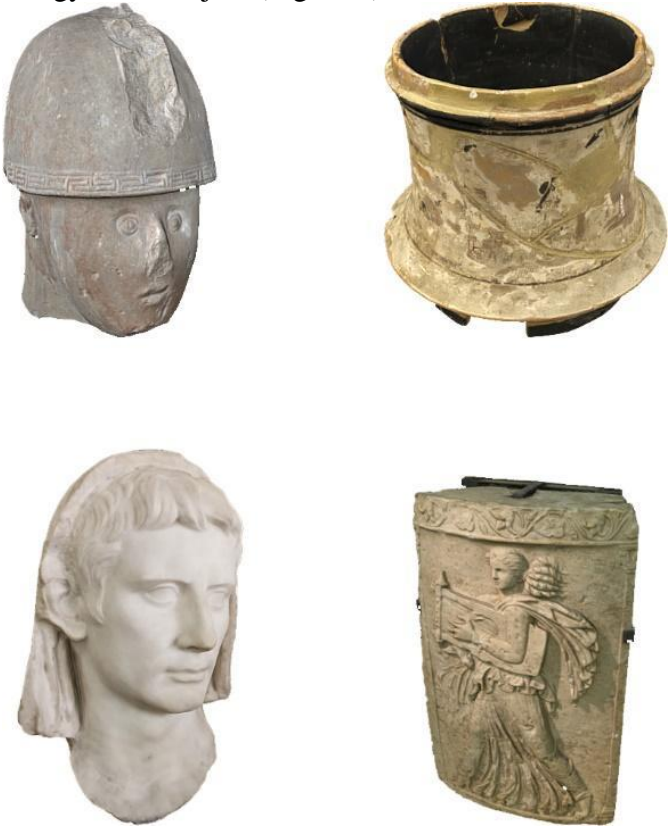

Figure 3. Some of the artefacts digitized by photogrammetry

The process structured in this way makes the acquisition very expeditious with a time for the photographic acquisition of about 20 minutes for each item. In the case of artefacts that cannot be easily moved from their showcases because of their dimensions pictures are taken moving around the object observing the same criteria of distance, overlap between consecutive pictures and not forgetting to regard his morphology. This method is less fast and accurate than semi-automatic one both in the acquisition phase and in the model creation phase because the dense point cloud must be cleaned from the object and space around the artefact.

\subsection{Create a virtual space. Two different case studies}

Potential of VR may be applied to existing sites and architectures as well to recreate no more existing environments. Here are presented two different cases studies: the National Archaeological Museum of Marche in Ancona and the 3D reconstruction of the Roman Forum of Fanum Fortunae.

3.2.1 The National Archaeological Museum of Marche in Ancona: Spherical panorama images (Figure 4) make possible the construction of virtual environments from a real existing one in a very expeditious way.

A series of pictures are taken from the same point of view with a 360-degree horizontal axis rotation and a 180-degree vertical axis rotation. Repeating this acquisition changing more than fifty points of view inside the rooms of the National Archaeological Museum of Marche in Ancona the entire exposition has been recreated. Single photos has been taken using a wide-angle lens in order to make also more expeditious the acquisition taking a smaller number of pictures and taking into account that we didn't need an high level of detail in the panorama but only the perception of the space. Passing from a panorama to another visitors explore the museum like if they are moving from one room to the next one.

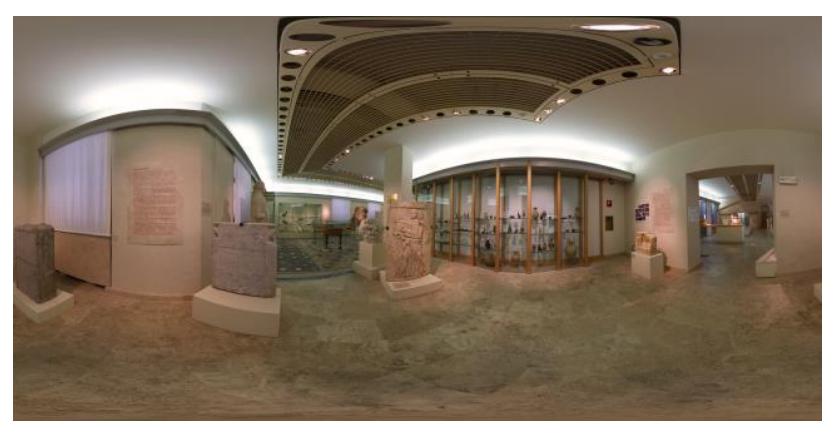

Figure 4. Spherical panorama of an expositive room

3.2.2 The 3D reconstruction of the Roman Forum of Fanum Fortunae: The modellation of this 3D reconstruction was made in the context of ArcheoFano Project that allowed for the first time a structured systematization of all archaeological data concerning the urban centre of the city of Fano (Figure 5). Data from laser scanner surveys, reconstructive hypotheses based on the study of Vitruvius De Architectura and examples of coeval architecture in the middle Adriatic region were integrated and in particular the reconstruction of the Basilica, that has never been found, was based on what Vitruvio wrote in the book V of De Architectura taking also in consideration the numerous illustrations made by various scholars.

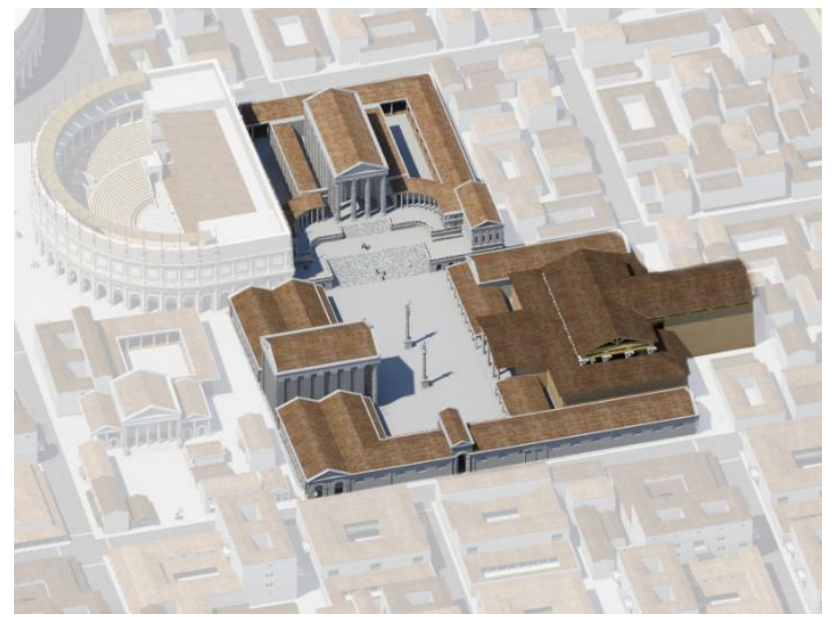

Figure 5. The Roman Forum in the context of the 3D reconstruction of the ancient Fanum Fortunae 
The use of a 3D model instead of spherical panoramas implies also the issue of real-time realistic visualization of virtual spaces. While a static photorealistic render (

Figure 6) sometimes can take hours of computational time, for VR application this cannot be possible because it's necessary a real-time rendering. Big and complex models are hardly handled by common computers so they have to be optimized to make an easier and faster processing and to make a more fluid and natural visualization constrained by the graphics card performance. Therefore, the number of the vertices of the model has to be reduced in order to make the file size smaller. The negative aspect of this operation is that reducing the number of the polygons of a model, it starts not to be photorealistic anymore, that is why also an optimization of the textures is necessary.

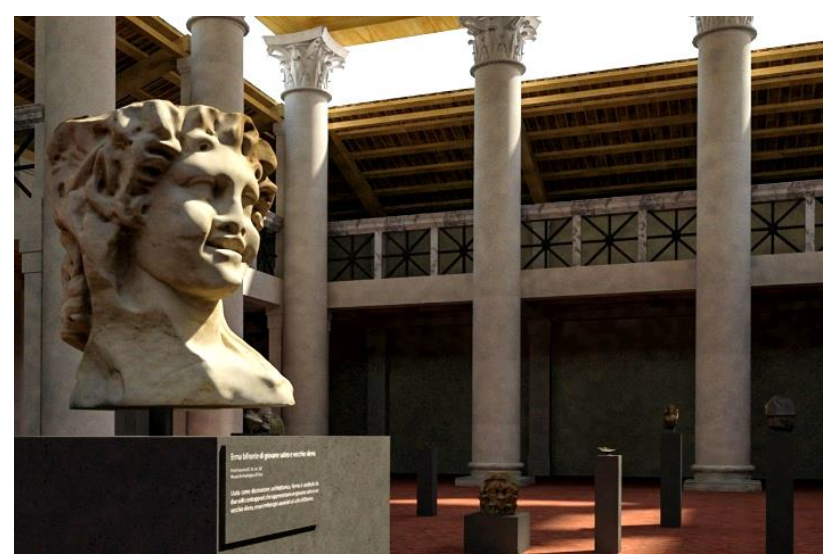

Figure 6. Still render of the reconstruction of the Roman Basilica

\subsection{Optimization for virtual interaction}

The two applications here presented show two different typologies of three-dimensional models: reality-based models of the artefacts collected in the museum and the 3D reconstruction of the lost architectures. Both were high poly and detailed models: the first one were created using Close Range Photogrammetry and Structure from Motion for the study, the documentation and the preservation of the artefacts, while the virtual reconstructions were made to have an accurate scientific reconstruction and still photo-realistic renders.

The pipeline to reduce the number of the polygons of the model without losing the visual quality for the user experience is made by three main steps: the decimation of the model, the unwrapping and finally the projection and baking of the maps.

While the second and third steps are the same for both kind of models, the decimation is made in two different ways. The 3D models of the artefacts, reality-based models digitized by photogrammetry, are decimated in Geomagic Studio, a specific software for the editing of point clouds and 3D models. It has been chosen this software instead of managing the entire process of the optimization inside 3ds Max (we could choose the "ProOptimizer" tool in 3ds Max, a tool to change from high to low resolution models) because here the decimation can be controlled better with many parameters: we have chosen a curvature priority decimation in order to preserve more details where the curvature is higher. Moreover sometimes the $3 \mathrm{ds}$ Max tool has problems with managing models with too many polygons. In fact, we decided to make models with the highest resolution in order to see all the smallest details typical of archaeological artefacts and later to decimate them for the VR application.

For the 3D reconstruction of the Roman Forum of Fanum Fortunae the decimation is made by CAD modelling in $3 \mathrm{ds}$ Max the most geometrically complex elements of the scene and deleting manually all the faces that could not be seen by the user inside the app (top of the roof, bottom of the pavements etc.). The decimation for the finds in addition to be faster is also higher than the decimation of the model of the Roman Forum (Table 1).

This operation is extremely slower than the decimation of the artefacts because is not automated but the starting point was a CAD model and automated decimation for this kind of models produces geometric errors like self-intersections, holes, flipped normals.

Once the geometry of the model is simplified reducing the number of the polygons, the UV coordinates has to be generated for each vertex in the mesh with the so called unwrapping operation. For each element an UV map is created trying to fill as much as possible the square map in order to have less wasted space and to save a smaller texture.

The third and last step is the projection of the geometric and visual characteristics of the high poly models on the low poly

\begin{tabular}{|l|c|c|}
\hline $\begin{array}{l}\text { Num. of polygons of the } \\
\text { original model }\end{array}$ & ca 3 millions & ca 9 millions \\
\hline $\begin{array}{l}\text { Size of the original model } \\
\text { (.obj format) }\end{array}$ & $600 \mathrm{MB}$ & $700 \mathrm{MB}$ \\
\hline $\begin{array}{l}\text { Num. of polygons of the } \\
\text { optimized model }\end{array}$ & ca 3000 & ca 800000 \\
\hline $\begin{array}{l}\text { Size of the optimized } \\
\text { model (.obj format) }\end{array}$ & $200 \mathrm{~KB}$ & $92 \mathrm{MB}$ \\
\hline
\end{tabular}

Table 1. Number of polygon and size of the models before and after the decimation for the virtual interaction

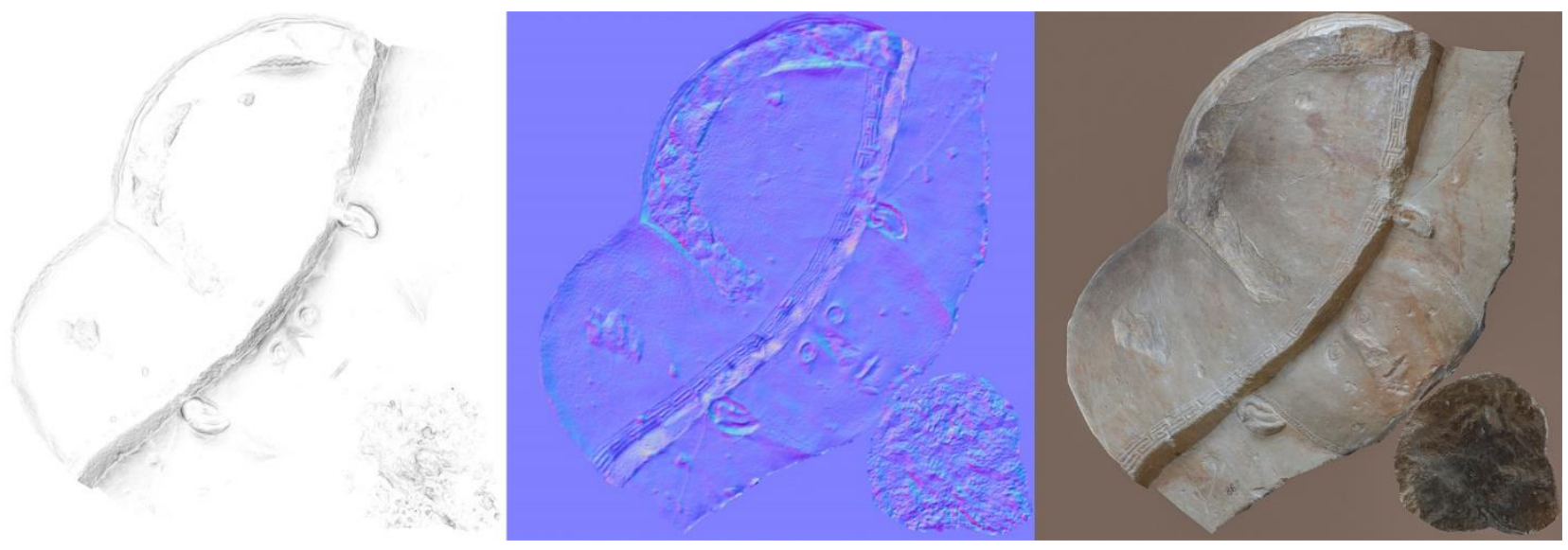

Figure 7. Ambient Occlusion map, Normal map and Diffuse map of one of the digitized artefacts 
ones. This is essential to make the user believe that he is looking at a detailed model. This operation is made in 3ds Max using the "Projection" modifier: the low and high poly models are positioned at the same point so that they are coincident, the "projection" modifier is activated on the low poly model, the high poly one is picked as reference geometry and a cage for the projection is created.

At this point all the information we want to project to the low poly model can be baked with the "Render to Texture" tool in $3 \mathrm{ds}$ Max. We create three maps: a normal map that simulate the lighting of bumps and dents, used to add details without using more polygons, an ambient occlusion (AO) map that calculate how exposed each point in a scene is to ambient lighting and a diffuse map that represent the color texture (Figure 7).

Once imported in the software for the development of the VR app the lowpoly decimated model appears very draft without maps (Figure 8a e b) but when Normal and AO maps (Figure 8c) and Diffuse map (Figure 8d) are applied it looks like the highpoly one.

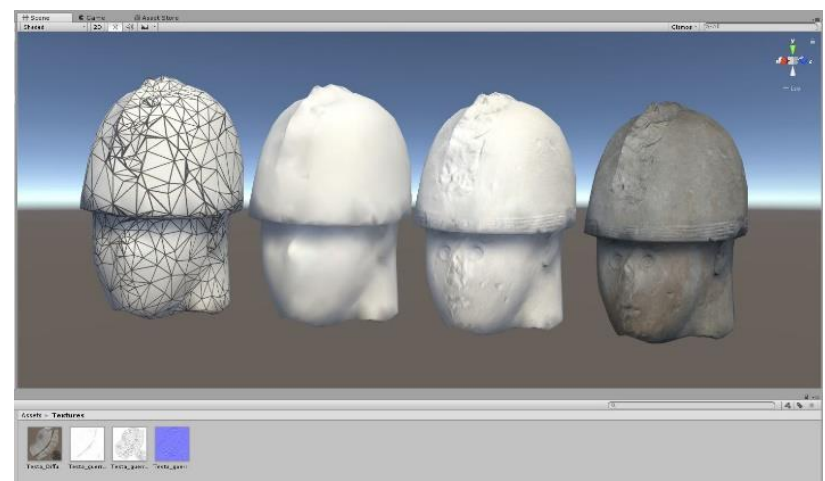

Figure 8 . The lowpoly model in wireframe visualization (a), shaded without any maps (b), with Normal and Ambient Occlusion maps (c) and finally with the two previous maps and the Diffuse one (d)

\section{IMMERSIVE REALITY. INTEGRATION OF CONTENTS AND CONTAINER}

Once that contents (artefacts models) and containers (spherical panoramas and 3D model) are created, they are integrated in the Unity game engine, a game development platform that allows to build $3 \mathrm{D}$ and 2D games, deploy them across mobile, desktop, VR/AR, consoles or the Web. The models are exported from 3ds Max in .fbx format file, that permit to export not only 3D object with their materials and textures but also lights, animations and cameras. In Unity are developed the interactions between the user and the models inside a 3D space.

The immersive fruition of the integrated data is possible thanks to a PC with a HTC Vive system and a monitor. Composed by a head-mounted display with a camera near the bottom rim, two wireless motion tracked handheld controllers and two "lighthouse" basestations for "room scale", HTC Vive system allows to replicate the natural movements of the user's body and to interact with the 3D models displaced in the virtual reality (Figure 9).

The headset thanks to features like a resolution of $1080 \times 1200$ pixels per eyes, a field of view of 110 degrees and a SteamVR Tracking to find the location of each sensor, a G-sensor, a gyroscope and proximity sensor is a very powerful and valuable device for VR applications. The use of two combined basestations, essentially two-multi axis laser emitter, guarantees 360 degrees coverage while the two controllers allow the interaction with things inside the virtual space making possible to grab, to handle and so to look up close at the objects.
These interactions are developed in Unity, where is possible to give specific commands to each button of the controllers. Here are also implemented the artefacts behaviour: they can be handled using the Rigidbody component, the main component that enables physical behaviour for an object. With a Rigidbody attached, the object will immediately respond to gravity, it can receive forces to make your objects move in a realistic way (Figure 10).

This potential are exploited as a valuable aid to enrich users experience and learning storage. The virtual space can be structured like a learning path that leads the visitor following a specific storytelling.

The user navigation inside the virtual space is always guided. In the Archaeological Museum application, thanks to buttons combination of the HTC controllers, it is possible to recall a map of the building that shows where the visitor is, allows to switch the panorama changing the point of view inside a room or the room itself. The virtual space presents also different kinds of hotspot. Position hotspot leads you to the next panorama whereas objects hotspot let you grab the object itself or visualize historical information about it (Figure 11).

The main difference for the final user in the Roman Forum application is that movements, as explained above, are totally free. The SteamVR Tracking Basestations sweep the room with multiple sync pulses and laser lines, reaching out to about 5 meters. By keeping careful track of the timings between pulses and sweeps, the SteamVR Tracking system uses simple trigonometry to find the location of each sensor to within a fraction of a millimeter. By combining multiple sensors, 2 basestations, as well as adding a high speed IMU (inertial measurement unit), SteamVR also calculates the tracked object's orientation, velocity, and angular velocity, all at an update rate of $1000 \mathrm{~Hz}$. So loading a 3D space reconstruction, like the one carried out for the Roman Forum of Fanum Fortunae, instead of a 2D panorama, the HTC Vive system is able to let the user move inside the virtual space as he is moving in the real one. The space limits determined by the field of view of the basestations, about $5 \times 5$ meters, is overwhelmed by the possibility of using controllers as laser pointers to get any visible point of the virtual space, after you reach that point the coordinate system of the virtual space are recalculated around the new origin as well as the new space limits (Figure 12).

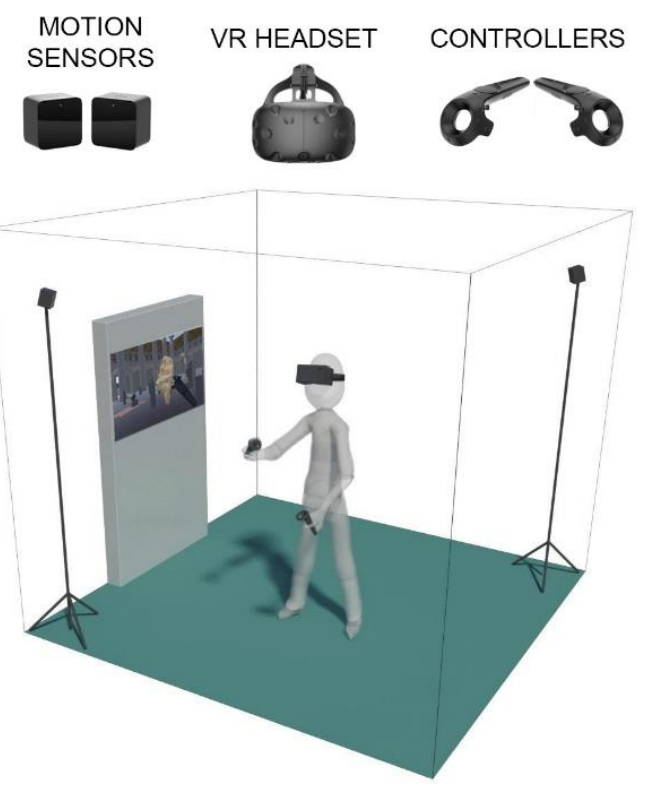

Figure 9. HTC Vive system for virtual interaction 


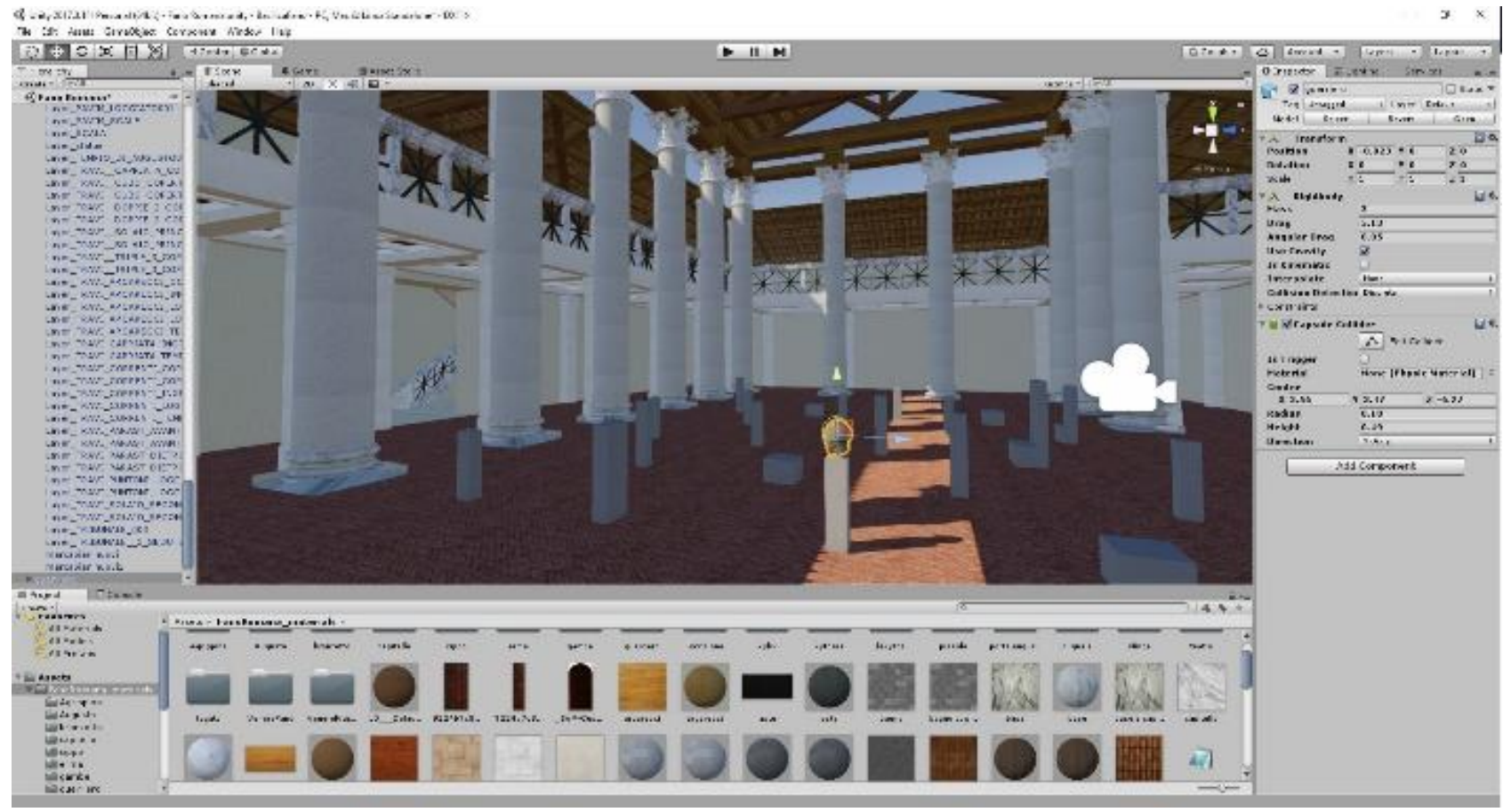

Figure 10. Development inside Unity of the interaction between the user and the 3D artefact situated inside the Basilica

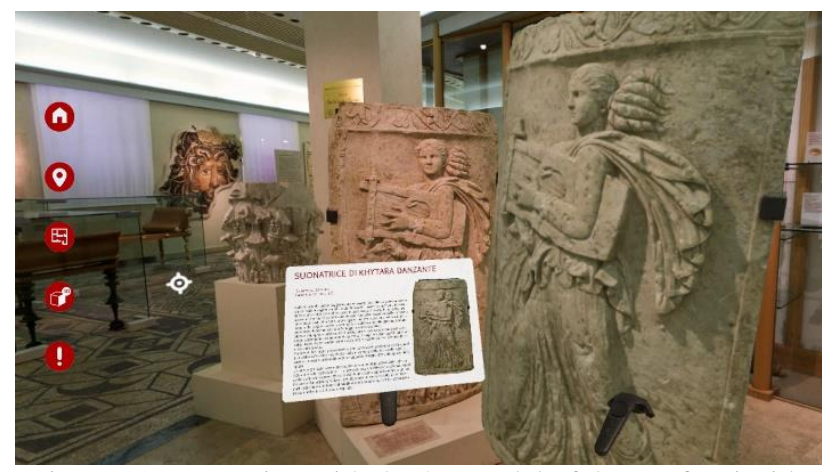

Figure 11. Interaction with the 3D model of the artefact inside the spherical panorama

\section{CONCLUSIONS AND RESULTS}

This paper presents a methodology based on a VR system exploited to visualize and interact with reality-based 3D models in an immersive virtual reality. This potential allows disseminating the knowledge preserved in museums in an innovative and appealing way involving the user in a fun and exciting experience. Once the artefacts are digitized and optimized the use of the software Unity ensures the integration with the high resolutions panoramic images or 3D model reconstructions that will represent the virtual space. Unity allows also creating intuitive user interfaces and this is central for an application that will be used not only by insiders like historians or archaeologists but also by tourists and students. The VR experience becomes a guided walk through museums and archaeological sites overcoming distances and time. Different purposes can be achieved offering a very immersive realism and a possibility of interaction otherwise inconceivable; temporary exhibits can be visited also after their deadline, no longer existing sites or architecture can be explored. Hypothetical reconstructions and cultural information structured on different levels can also be added as layers making this fruition system also a database for several kind of information available for researchers anytime and anywhere.

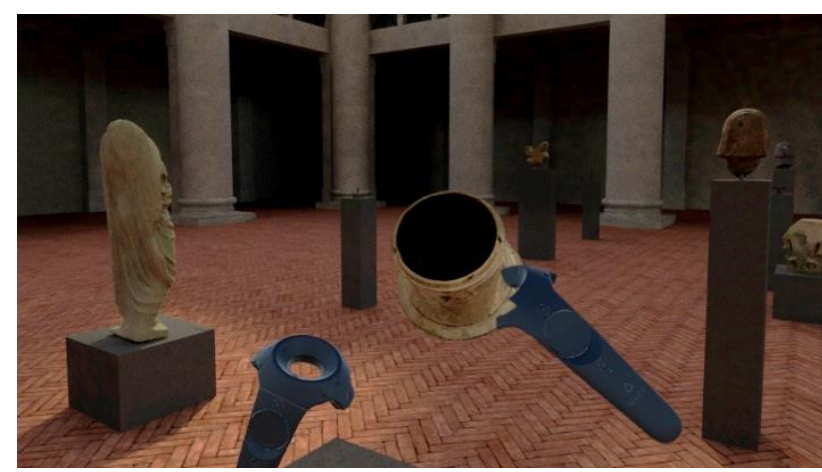

Figure 12. Interaction with the 3D model inside virtual space of the Basilica reconstruction

\section{REFERENCES}

Anthes, C., Garcia-Hernandez, R.J., Wiedemann, M., Kranzlmuller, D., 2016. State of the art of virtual reality technology, in: 2016 IEEE Aerospace Conference. pp. 1-19. https://doi.org/10.1109/AERO.2016.7500674

Boas, Y., 2013. Overview of Virtual Reality Technologies. Mms.Ecs.Soton.Ac.Uk.

Clini, P., Frapiccini, N., Mengoni, M., Nespeca, R., Ruggeri, L., 2016. SFM technique and focus stacking for digital documentation of archaeological artifacts. Int. Arch. Photogramm. Remote Sens. Spat. Inf. Sci. - ISPRS Arch. 41, 229-236. https://doi.org/10.5194/isprsarchives-XLI-B5-2292016

Demetrescu, E., Ferdani, D., Dell'Unto, N., Touati, A.-M.L., Lindgren, S., 2016. Reconstructing the original splendour of the House of Caecilius Iucundus. A complete methodology for virtual archaeology aimed at digital exhibition. SCIRES-IT - Sci. Res. Inf. Technol. https://doi.org/10.2423/i22394303v6n1p51

Dostal, C., Yamafune, K., 2018. Photogrammetric texture 
mapping: A method for increasing the Fidelity of 3D models of cultural heritage materials. J. Archaeol. Sci. Reports 18, 430 436. https://doi.org/10.1016/j.jasrep.2018.01.024

Jiménez Fernández-Palacios, B., Morabito, D., Remondino, F., 2017. Access to complex reality-based 3D models using virtual reality solutions. J. Cult. Herit. 23, 40-48. https://doi.org/10.1016/j.culher.2016.09.003

EPOCH, 2009. The London Charter, Access.

Evgenikou, V., Georgopoulos, A., 2015. Investigating 3D reconstruction methods for small artifacts. Int. Arch. Photogramm. Remote Sens. Spat. Inf. Sci. - ISPRS Arch. 40, 101-108. https://doi.org/10.5194/isprsarchives-XL-5-W4-1012015
Gattet, E., Devogelaere, J., Raffin, R., Bergerot, L., Daniel, M., Jockey, P.H., De Luca, L., 2015. A versatile and low-cost 3D acquisition and processing pipeline for collecting mass of archaeological findings on the field. Int. Arch. Photogramm. Remote Sens. Spat. Inf. Sci. - ISPRS Arch. 40, 299-305. https://doi.org/10.5194/isprsarchives-XL-5-W4-299-2015

Lopez-Menchero, V.M., Grande, A., 2011. The Principles of the Seville Charter. Proc. XXIIIrd Int. CIPA Symp. 12-16.

Skamantzari, M., Georgopoulos, A., 2016. 3D Visualization for virtual museum development. Int. Arch. Photogramm. Remote Sens. Spat. Inf. Sci. - ISPRS Arch. 41, 961-968. https://doi.org/10.5194/isprsarchives-XLI-B5-961-2016 